\title{
Efecto de la frecuencia de aplicación de estrógenos locales sobre el grosor endometrial en mujeres posmenopáusicas
}

\author{
Sebastián Carranza-Lira, ${ }^{1}$ Yazmín del Carmen Culebro-Castro, ${ }^{2}$ Víctor Alberto Olguín-Cruces, ${ }^{3}$ \\ Sergio Rosales-Ortiz, ${ }^{4}$ Diana Sulvaran-Victoria ${ }^{5}$ y Elizabeth Sánchez-Merino ${ }^{3}$ \\ ${ }^{1}$ División de Investigación en Salud; ${ }^{2}$ Ex-residente de la especialidad de Ginecología y Obstetricia; ${ }^{3}$ Servicio de Anatomía Patológica; ${ }^{4}$ Servicio de \\ Ultrasonido; ${ }^{5}$ Servicio de Ginecología Endocrina. Instituto Mexicano del Seguro Social, Hospital de Hospital de Gineco-Obstetricia 4 "Luis Catelazo \\ Ayala", Ciudad de México, México
}

\begin{abstract}
Resumen
Introducción: Los estrógenos conjugados vía vaginal para aliviar la atrofia y sequedad vaginales pueden producir cambios endometriales. Objetivo: Conocer el efecto de la frecuencia de aplicación de estrógenos conjugados vía vaginal en el grosor endometrial en mujeres posmenopáusicas. Método: Se estudiaron 70 mujeres posmenopáusicas con sequedad vaginal que recibieron estrógenos conjugados en crema $(0.625 \mathrm{mg} / 1 \mathrm{~g})$ durante 12 semanas divididas de la siguiente manera según la frecuencia de aplicación: grupo 1 , dos veces por semana $(n=35)$ y grupo 2 , tres veces por semana $(n=35)$. Al inicio y final del tratamiento se determinó el valor estrogénico en la citología vaginal y se realizó ultrasonido endovaginal para medir el grosor endometrial. La comparación entre los grupos se realizó con $U$ de Mann-Whitney y entre los valores pre y postratamiento con prueba de Wilcoxon. Resultados: De 70 mujeres reclutadas solo se estudiaron 38 mujeres, 19 en cada grupo, pareadas por valor estrogénico inicial. No se encontró diferencia entre los grupos, ni antes ni después del tratamiento, en el índice de maduración, valor estrogénico ni grosor endometrial. Conclusión: No hubo diferencias en el grosor endometrial entre las distintas frecuencias de aplicación de estrógenos conjugados en crema.
\end{abstract}

PALABRAS CLAVE: Grosor endometrial. Estrógenos conjugados. Frecuencia de aplicación.

\begin{abstract}
Introduction: Conjugated estrogens, when used by the vaginal route for the relief of vaginal dryness and atrophy, can produce endometrial changes. Objective: To know the effect of vaginal conjugated estrogens application frequency on endometrial thickness in postmenopausal women. Method: Seventy postmenopausal women with vaginal dryness who received conjugated estrogen cream $(0.625 \mathrm{mg} / 1 \mathrm{~g})$ for 12 weeks were studied. The women were divided according to application frequency as follows: group 1, twice-weekly $(n=35)$, and group 2, thrice-weekly $(n=35)$. At baseline and at end-of-treatment, vaginal cytology was examined to determine the estrogenic value, and an endovaginal ultrasound was performed to measure endometrial thickness. The comparison between groups was carried out with Mann Whitney's U-test, and the comparison between baseline and post-treatment values, with Wilcoxon's test. Results: Of 70 recruited women, only 38 were studied, 19 in each group, paired by baseline estrogenic value. No difference was found between groups, neither at baseline nor after treatment, in the maturation index, estrogenic value or endometrial thickness. Conclusion: There were no differences in endometrial thickness between the conjugate estrogen cream different application frequencies.
\end{abstract}

KEY WORDS: Endometrial thickness. Conjugated estrogens. Application frequency.

Correspondencia:

Sebastián Carranza-Lira

E-mail: drsebastiancarranza@gmail.com
Fecha de recepción: 24-09-2018

Fecha de aceptación: 24-01-2019

DOI:10.24875/GMM.19004706
Gac Med Mex. 2019;155:199-201

Disponible en PubMed www.gacetamedicademexico.com 


\section{Introducción}

El climaterio es el periodo que antecede y sigue al cese de la menstruación, en él se presentan alteraciones bioquímicas y clínicas ocasionadas por la disminución de las funciones ováricas en la producción hormonal y de gametos. ${ }^{1}$ El epitelio vaginal se compone de varias capas de células, las cuales se modifican por la deficiencia de estrógenos. ${ }^{2}$

Es posible valorar el grado de estrogenización del epitelio vaginal por medio del índice de maduración, el cual se obtiene de la relación porcentual de tres tipos de células vaginales (parabasales/intermedias/ superficiales) y mediante el cual se calcula el valor estrogénico. En la posmenopausia temprana predominan las células intermedias y en la tardía, las células parabasales. ${ }^{3}$ La terapia estrogénica local mejora las condiciones de atrofia y sequedad vaginal, al incrementar el porcentaje de células superficiales. ${ }^{2,4}$

En el endometrio, el uso de estrógenos aumenta la actividad mitótica desencadenando proliferación. ${ }^{5,6} \mathrm{El}$ endometrio posmenopáusico, expuesto al estrógeno sin oposición, debe ser vigilado estrechamente, lo que es posible con la medición ultrasonográfica de su grosor. $^{7-12}$ En diversos estudios se ha comparado la ecografía transvaginal con biopsia endometrial para la detección de patología endometrial; encontraron que el ultrasonido tiene valor predictivo negativo de $99 \%$, sensibilidad de $90 \%$, especificidad de $48 \%$ y valor predictivo positivo de $9 \%$ para la detección de anormalidad, además, es menos doloroso e incómodo para la paciente y más accesible. ${ }^{12-14}$

Para evitar el riesgo de hiperplasia endometrial con el uso de estrógenos locales por vía vaginal parece prudente utilizarlos con menor frecuencia, por ejemplo, únicamente dos veces por semana en vez de tres.

El objetivo del presente estudio fue determinar el efecto de dos distintas frecuencias de aplicación de estrógenos conjugados locales en el endometrio de mujeres posmenopáusicas.

\section{Método}

Estudio prospectivo, longitudinal, comparativo, abierto, controlado, en el que se estudiaron mujeres posmenopáusicas atendidas en un servicio de ginecología endocrina. Todas debían tener por lo menos un año desde la menopausia y referir atrofia y sequedad vaginal. No fueron incluidas las mujeres con tratamiento hormonal o patología que contraindicara el uso de estrógenos. En todas se documentó edad
Tabla 1. Datos generales en dos grupos de mujeres posmenopáusicas que recibieron estrógenos conjugados

\begin{tabular}{llll}
\hline Variable & \multicolumn{1}{c}{ Grupo 1 } & & \multicolumn{1}{c}{ Grupo 2 } \\
\cline { 2 - 2 } & Media (rango) & & Media (rango) \\
\hline Edad (años) & $58(49-72)$ & & $59(51-78)$ \\
Peso (kg) & $70(50-97)$ & & $60.5(50-104)$ \\
Talla (m) & $1.57(1.42-1.64)$ & & $1.49(1.45-1.68)$ \\
Índice de masa corporal & $28.8(18.6-39.8)$ & & $27.1(21.8-48.0)$ \\
Perímetro de cintura (cm) & $94(78-115)$ & & $92(80-121)$ \\
Perímetro de cadera (cm) & $100(89-118)$ & & $99(94-115)$ \\
Índice cintura-cadera & $0.93(0.80-1.15)$ & $0.92(0.83-1.12)$ \\
\hline Grupo 1, estrógenos conjugados vía vaginal 0.625 mg dos veces por semana. \\
Grupo 2, estrógenos conjugados vía vaginal 0.625 mg tres veces por semana.
\end{tabular}

(años), peso $(\mathrm{kg})$, talla $(\mathrm{m})$ e índice de masa corporal (IMC, peso en $\mathrm{kg} /$ talla en $\mathrm{m}^{2}$ ) asimismo, se documentó el perímetro de la cintura $(\mathrm{cm})$ y de la cadera $(\mathrm{cm})$ y se calculó el índice cintura-cadera (perímetro de la cintura/perímetro de la cadera).

En las mujeres que aceptaron participar, antes del inicio del tratamiento se tomó una citología vaginal en la cual se determinó el índice de maduración y el valor estrogénico, ${ }^{3}$ además, se realizó un ultrasonido transvaginal en el que se midió el grosor endometrial. ${ }^{7}$ Todas las interpretaciones citológicas fueron efectuadas por el mismo médico, al igual que los estudios ultrasonográficos.

Todas las mujeres recibieron estrógenos conjugados en crema $(1 \mathrm{~g} / 0.625 \mathrm{mg})$ durante tres meses y según la frecuencia de aplicación fueron incluidas en uno de dos grupos:

- Grupo 1: dos veces por semana.

- Grupo 2: tres veces por semana.

Al término del tratamiento se les realizó una nueva citología vaginal y se midió el grosor endometrial.

Considerando un intervalo de confianza de $95 \%$, una potencia de $90 \%$, una relación de expuestos a no expuestos de 1 a 1, una frecuencia estimada de cambio de $40 \%$ en el grupo con aplicación dos veces por semana y $80 \%$ en el de tres veces por semana resultó un tamaño de muestra de 34 pacientes por grupo.

La comparación entre los grupos se realizó con U de Mann-Whitney y la comparación entre los valores pre y postratamiento con prueba de Wilcoxon.

El protocolo fue autorizado por el Comité Local de Investigación y el Comité de Ética en Investigación con el registro R-2016-3606-49.

\section{Resultados}

De las 70 mujeres, 35 quedaron en el grupo 1 y completaron el tratamiento 33; en el grupo 2, de las 35 
Tabla 2. Valores iniciales y finales en los parámetros de la citología vaginal y en el grosor endometrial en dos grupos de mujeres posmenopáusicas que recibieron estrógenos conjugados

\begin{tabular}{llll}
\hline Momento & Parámetro & \multicolumn{1}{c}{ Grupo 1 } & \multicolumn{1}{c}{ Grupo 2 } \\
\hline Inicial & Parabasales (\%) & $100(0-100)^{\mathrm{a}}$ & $100(0-100)^{\mathrm{A}}$ \\
& Intermedias (\%) & $0(0-100)^{\mathrm{b}}$ & $0(0-100)^{\mathrm{B}}$ \\
& Superficiales (\%) & $0(0-5)^{\mathrm{C}}$ & $0(0-5)^{\mathrm{C}}$ \\
& Valor estrogénico & $0(0-52.5)^{\mathrm{d}}$ & $0(0-52.5)^{\mathrm{D}}$ \\
& Grosor endometrial $(\mathrm{mm})$ & $2.2(1.4-3.1)^{\mathrm{e}}$ & $2.2(0.82-3.0)$ \\
Final & Parabasales (\%) & $10(0-100)^{\mathrm{a}}$ & $0(0-100)^{\mathrm{A}}$ \\
& Intermedias (\%) & $85(0-100)^{\mathrm{b}}$ & $90(0-100)^{\mathrm{B}}$ \\
& Superficiales (\%) & $0(0-10)^{\mathrm{C}}$ & $2.5(0-60)^{\mathrm{C}}$ \\
& Valor estrogénico & $45(0-55)^{\mathrm{d}}$ & $51.2(0-80)^{\mathrm{D}}$ \\
& Grosor endometrial $(\mathrm{mm})$ & $2.7(1.2-3.4)^{\mathrm{e}}$ & $2.4(0.8-3.6)$ \\
\hline
\end{tabular}

Grupo 1, estrógenos conjugados vía vaginal $0.625 \mathrm{mg}$ dos veces por semana.

Grupo 2, estrógenos conjugados vía vaginal $0.625 \mathrm{mg}$ tres veces por semana.

Valores de $\mathrm{p}$ :

${ }^{\mathrm{a}}<0.043,{ }^{\mathrm{b}}<0.065,{ }^{\mathrm{C}}<0.063,{ }^{\mathrm{d}}<0.016,{ }^{\mathrm{e}}<0.029$

${ }^{\mathrm{A}}<0.010,{ }^{\mathrm{B}}<0.022,{ }^{\mathrm{C}}<0.018,{ }^{\mathrm{D}}<0.009$

mujeres solo 30 completaron el tratamiento. Al comparar los grupos a pesar de que las mujeres fueron incluidas en cada grupo alternadamente, se encontró que existían diferencias entre ambos en el valor estrogénico inicial. Por ese motivo fueron pareadas por el valor estrogénico inicial, quedando 38 mujeres, 19 en cada grupo (potencia $80 \%$ ); 17 y 16, respectivamente en los grupos 1 y 2 , completaron el tratamiento.

No se encontró diferencia entre los grupos en edad, peso, talla, IMC, perímetro de la cintura, perímetro de la cadera e índice cintura-cadera (Tabla 1).

Al comparar ambos grupos no hubo diferencias estadísticamente significativas en el porcentaje de células parabasales, intermedias y superficiales, tampoco en el índice de maduración, valor estrogénico o grosor endometrial, ni antes ni después del tratamiento (Tabla 2).

Al comparar cada grupo por separado, los valores basales y finales, en el grupo 1 hubo disminución significativa del porcentaje de células parabasales e incremento del valor estrogénico posterior al tratamiento. Asimismo, el grosor endometrial se incrementó significativamente.

En el grupo 2 hubo disminución significativa del porcentaje de células parabasales e incremento en el de intermedias y superficiales. Asimismo, el valor estrogénico se incrementó significativamente. No hubo cambio significativo en el grosor endometrial (Tabla 2).

\section{Discusión}

En este estudio, tanto la aplicación dos veces como tres veces por semana, condicionó un incremento en el valor estrogénico, lo que traduce que ambas dosis son suficientes para mejorar el trofismo vaginal. 2,4,6

El endometrio prolifera con la aplicación de estrógenos, ${ }^{2}$ pero en el estudio no se encontraron diferencias entre los grupos en cuanto al grosor endometrial, lo cual traduce que inducen los mismos cambios. En un estudio de revisión se reportó que la administración vaginal de estrógenos provoca incremento no significativo del grosor endometrial, ${ }^{15}$ sin embargo, en el análisis de cada grupo se encontró que solo en el grupo con aplicación dos veces por semana el incremento fue significativo con $22.7 \%$, mientras que en el grupo con aplicación tres veces por semana fue de $9 \%$, para lo cual no tenemos explicación. Cabe mencionar que el grosor estuvo dentro del "rango de seguridad" .,11

Con base en lo anterior, es posible concluir que ambas frecuencias de aplicación condicionan mejoría en el ámbito vaginal sin mayor compromiso del endometrio.

\section{Bibliografía}

1. Carranza-Lira S. Introducción a la endocrinología ginecológica. México: Trillas; 2011.

2. Carranza-Lira S, Cabrera-López TJ, Estrada Moscoso I, Aguado-Pérez RA. Índice de maduración y sequedad vaginales. Evaluación de dos dosis de estrógenos tópicos vía vaginal. Rev Med Inst Mex Seguro Soc. 2012;50:315-318.

3. Carranza-Lira S, Amador-Pérez C, MacGregor-Gooch AL. Índice de maduración y sequedad vaginales en la postmenopausia con uso de calcio y calcitriol. Rev Med Inst Mex Seguro Soc. 2012;50:537-540.

4. Suckling J, Lethaby A, Kennedy R. Local oestrogen for vaginal atrophy in postmenopausal women. Cochrane Database Syst Rev. 2006;4: CD001500.

5. Carranza-Lira S, Martínez-Chéquer JC, Santa-Rita MT, Ortiz de la-Peña A, Pérez Y, Fernández RL. Endometrial changes according to hormone replacement therapy schedule. Menopause. 1998;5:86-89.

6. Archer DF. Efficacy and tolerability of local estrogen therapy for urogenital atrophy. Menopause. 2010;17:194-203.

7. Carranza-Lira S, Ortíz-Rodríguez ML, Martínez-Chéquer JC, Santa Rita-Escamilla MT, García-Hernández E, Romo-Aguirre C. Correlación de los hallazgos histopatológicos con las variables ultrasonográficas del endometrio según el peso y distribución del tejido adiposo en la posmenopausia. Ginecol Obstet Mex. 1996;64:517-521.

8. Ozer A, Ozer S, Kanat-Pektas M. Correlation between transvaginal ultrasound measured endometrial thickness and histopathological findings in Turkish women with abnormal uterine bleeding. J Obstet Gynecol Res. 2016:42:573-578.

9. Van-Hanegem N, Breijer MC, Khan KS, Clark TJ, Burger MPM, Mol BWJ, et al. Diagnostic evaluation of the endometrium in postmenopausal bleeding: An evidence-based approach. Maturitas. 2011;68:155-164.

10. Sit AS, Modugno F, Hill LM, Martin J, Weissfeld JL. Transvaginal ultrasound measurement of endometrial thickness as a biomarker for estrogen exposure. Cancer Epidemiol Biomarkers Prev. 2004;13:1459-1465.

11. Sheng Q, Yang J Zhao Q, Li F. Dynamic monitoring of menopause hormone therapy and defining the cut-off value of endometrial thickness during uterine bleeding. J Biomed Res. 2016;30:191-196.

12. Omodei U, Ferrazzia E, Ruggeri C, Palai N, Fallo L, Dordoni D, et al. Endometrial thickness and histological abnormalities in women on hormonal replacement therapy: a transvaginal ultrasound/hysteroscopic study. Ultrasound Obstet Gynecol. 2000;15:317-320.

13. Saha TK, Amer SA, Biss J, Thakare H, Williams S, Farrell CT, et al. The validity of transvaginal ultrasound measurement of endometrial thickness: a comparison of ultrasound measurement with direct anatomical measurement. BJOG. 2004:111:1419-1424.

14. Sturdee DW, Panay N. Recommendations for the management of postmenopausal vaginal atrophy. Climacteric. 2010;13:509-522.

15. Lethaby $A$, Ayeleke RO, Roberts $H$. Local oestrogen for vaginal atrophy in postmenopausal women. Cochrane Database Syst Rev. 2016;8: CD001500. 